\title{
PERFIL E TEMPO PORTA-BALÃO DE PACIENTES COM INFARTO AGUDO DO MIOCÁRDIO
}

\section{PROFILE AND BALLOON HOLDER TIME OF PATIENTS WITH ACUTE MYOCARDIAL INFARCTION}

Cladis Loren Kiefer Moraes

Magada Tessmann Rodrigo Valdir Botelho

Valter Mendonça Alice Schmitt Francieli Tozatti Ficagna

\section{INTRODUÇÃO}

Muito se tem discutido sobre as doenças cardiovasculares, estas doenças têm inúmeros agravos para o indivíduo, e podem levar a severas consequências. Estudos apontam que dentre as principais emergências atendidas, 13,59\% são emergências cardiovasculares, com resultados semelhantes para causas neurológicas $13,9 \%$, ficando apenas atrás das causas desconhecidas $23,47 \%{ }^{1}$.

O perfil epidemiológico está diretamente relacionado à síndrome coronariana aguda. Dados de estudos apontam que a idade, sexo, etnia, nível de escolaridade, comorbidades (Hipertensão Arterial Sistêmica, Diabetes, Dislipidemia) e o estilo de vida (sedentarismo, tabagismo, etilismo) estão associados diretamente ao infarto agudo do miocárdio ${ }^{2,3}$.

O enfoque do tempo resposta ao atendimento das emergências cardiológicas vem sendo cada vez mais priorizado, pois, com o menor tempo resposta melhor será o prognóstico do paciente. O momento do surgimento dos sintomas até a decisão de 
procurar atendimento é caracterizado pela demora, há uma demora na chegada dos pacientes ao serviço de emergência quando do início dos sintomas ${ }^{4}$.

$O$ tratamento do IAM, principalmente com supradesnivelamento do segmento ST (IAM-ST), é basicamente tempo-dependente, pois o objetivo do tratamento é a reperfusão da artéria ocluída o mais breve possível. No entanto, em 40 a $65 \%$ dos casos, o óbito por IAM ocorre na primeira hora de manifestação da doença e $80 \%$ dos casos ocorre nas primeiras 24 horas ${ }^{4,5}$. Segundo dados do DATASUS o Infarto agudo do miocárdio é primeira causa de mortes no País. A maior parte das mortes por Infarto Agudo do Miocárdio (IAM) ocorrem nas primeiras horas de manifestação da doença, e geralmente não são assistidas pelos médicos ${ }^{7}$.

Com a implantação da Rede de Atenção a Urgência e Emergência (RUE), o ministério da saúde visa diminuir o dano causado pelo IAM e toma medidas estratégicas a fim de expandir e consolidar as linhas de cuidado de atenção. Seu foco é aumentar o número das unidades coronarianas, ampliar o acesso à angiologia primária, implantar protocolos de transferência e transporte para agilização do atendimento - início rápido do tratamento de reperfusão coronariana imediata dos pacientes. Além de qualificar o atendimento ao infarto nas urgências pré-hospitalares (SAMU e UPA) e implementar a integração entre o diagnóstico pré-hospitalar e a conduta hospitalar e garantir o fornecimento de medicamentos essenciais ao tratamento do $\mathrm{IAM}^{8}$.

$\mathrm{O}$ atendimento ao paciente com infarto inicia no atendimento pré-hospitalar caracterizado pelo início dos sintomas (geralmente dor torácica aguda), até a decisão de procurar atendimento e definir de que forma será seu deslocamento até o hospital. A fase pré-hospitalar, em ambos os momentos (início dos sintomas e tomada de decisão) são caracterizadas pela demora, especialmente em pacientes idosos, do sexo feminino e com baixo fator sócio econômico. A atenção aos pacientes com IAM se deve principalmente a gravidade da doença. O Infarto Agudo do Miocárdio com Supradesnivelamento de Segmento ST (IAMCSST), ocorre quando umas das artérias coronárias se tornam totalmente ocluída. A região do miocárdio irrigada por aquela artéria é privada de suprimento sanguíneo provocando isquemia que pode evoluir para necrose tecidual ${ }^{3}$. 
Os sintomas mais comuns no IAM podem aparecer individualmente ou em conjunto. A dor ou desconforto torácico irradiando para membro superior esquerdo (MSE), seguido de sudorese ou dispneia é a principal sintomatologia $(59,62 \%)$, seguido de dor torácica irradiada para MSE e dor epigástrica $(36,54 \%)$ em poucos casos aparecerem apenas sudorese ou dispneia $(3,85 \%)^{9}$.

Em virtude da gravidade do IAMCSST e o dano que pode causar é necessária uma rápida identificação com o propósito de identificar a patologia em curso. Dentro dos hospitais, o enfermeiro é o primeiro contato do paciente, e deve ter a capacidade para avaliar os sinais e sintomas, realizar a triagem de forma adequada para com agilidade iniciar a assistência. A atuação do enfermeiro é primordial no sentido de agilizar o processo de trabalho visando o atendimento qualificado, identificando os sinais e sintomas do paciente com IAMCSST bem como os encaminhamentos e as intervenções assistenciais que se seguem. As intervenções priorizadas pelo enfermeiro devem incluir eletrocardiograma, monitorização cardíaca, coleta de enzimas cardíacas, instalação de oxigênio, realização da anamnese e do histórico breve, glicemia capilar e punção de acesso venoso periférico de grosso calibre. A dor torácica é um sintoma que pode ser ocasionado por diversos tipos de doenças, o que pode retardar o diagnóstico de IAM. Portanto os usos de protocolos utilizados nos serviços de urgência e emergência cardiológica servem para orientar e facilitar o diagnóstico de IAM, proporcionando agilidade e qualidade nas intervenções necessárias para o atendimento ao paciente ${ }^{9}$.

No atual cenário temos o IAMCSST uma das doenças cardiovasculares de maior gravidade. $O$ atendimento prestado pela equipe de saúde das emergências é determinante para o curso da doença e gera efeitos benéficos para o paciente. Assim, identificar em uma emergência cardiológica o tempo entre o atendimento na emergência e a reperfusão coronariana fornece dados sobre o processo de trabalho de toda a equipe de saúde e permite rever os processos de trabalho em vigor a fim de adequá-los ou implementá-los. Além disso, a pesquisa proporcionará dados comparativos entre o tempo preconizado pela Sociedade Brasileira de Cardiologia e o tempo efetivo da emergência cardiológica pesquisada. Para tanto se tem como pergunta da pesquisa: Qual o perfil epidemiológico e o tempo resposta porta-balão dos pacientes com supradesnível de segmento ST atendidos na emergência? Para 
tanto, a pesquisa tem com objetivo identificar em um serviço especializado em cardiologia o tempo porta balão dos pacientes com síndrome coronariana aguda especificamente os pacientes de infarto com supradesnivelamento de segmento ST e o perfil epidemiológico e comorbidades deste paciente.

\section{MÉTODOS}

\section{Tipo de estudo}

Quanto à abordagem Metodológica realizou-se de uma pesquisa quantitativa do tipo documental, retrospectiva, aplicada, exploratória e de campo.

Local de estudo e coleta de dados; critérios de inclusão e exclusão.

A pesquisa foi realizada em uma Instituição Pública de referência em Cardiologia no estado de Santa Catarina. Os dados foram coletados a partir de informações contidas no prontuário eletrônico do paciente nos meses de abril a maio de 2017. Para a coleta de dados foi elaborado um instrumento pelos pesquisadores para caracterizar o perfil epidemiológico (variáveis demográficas: sexo, idade, raça, estado civil e socioeconômico: escolaridade), as comorbidades e fatores de risco para doença coronariana: HAS, dislipidemia, obesidade e uso de álcool e fumo e o tempo resposta porta balão e o tempo de início da dor e a chegada à emergência. Os critérios diagnósticos de IAMCSST foram os preconizados pelas diretrizes nacionais e internacionais.

Os critérios de inclusão foram: ser paciente atendido na emergência do Instituto de cardiologia de Santa Catarina nos anos de 2015 e 2016, ter diagnóstico médico de IAM com supra e angioplastia primária. Os critérios de exclusão são: não ser paciente com diagnóstico médico de IAM com supra, e com atendimento fora do recorte temporal.

Análise dos resultados

Os dados foram analisados no programa STATA 11.0. Inicialmente foram obtidas as frequências absolutas e relativas da distribuição da amostra para cada categoria das variáveis estudadas. Em seguida, foi realizada a verificação do Delta $T$ de entrada no Centro de Referência até o Início da Angioplastia, como também, a 
associação do tempo porta-balão com características, fatores de risco, comorbidades e a média do tempo porta-balão através da aplicação do Teste T (Student) nãopareado.

\section{Aspectos Éticos}

A pesquisa respeitou os aspectos éticos legais conforme Resolução no 466/12 do Conselho Nacional de Saúde ${ }^{10} \mathrm{e}$ foi aprovada pelo Comitê de Ética em pesquisa sob CAAE: 65575917.2.0000.0113.

\section{RESULTADOS}

Durante o período do estudo, 280 pacientes foram admitidos consecutivamente motivados por infarto agudo do miocárdio com Supradesnível do Segmento ST e com indicação de reperfusão.

Ao compararmos a distribuição dos pacientes infartados por faixa etária em relação ao sexo, podemos observar como mostra a Tabela 1, que a maior incidência de pacientes infartados do sexo masculino ocorreu na faixa etária dos 54 a 64 anos (31,07\%); já a do sexo feminino entre 65 e 76 anos (8,57\%). Nossos dados, apesar de se referirem a um grupo hospitalar restrito, também evidenciaram, a exemplo dos estudos pioneiros de Framingham, que no sexo masculino essa doença se manifestou aproximadamente 10 anos antes que no sexo feminino ${ }^{11}$.

Procedendo à caracterização do grupo estudado, encontramos uma maior incidência de IAM em pacientes de cor branca $(95,71 \%)$, casados $(58,21 \%)$, com idade entre 54 a $64(39,29 \%)$ e com ensino fundamental incompleto $(55,71 \%)$. 
Tabela 1 - Características Sócio demográficas dos pacientes com Diagnósticos de Infarto Agudo do Miocárdio com Supra de segmento ST, Florianópolis-SC, Brasil, 2015 e 2016

\section{Características}

$\mathrm{n}=\mathbf{2 8 0}(\%)$

\section{Sexo}

Masculino

$216(77,14)$

Feminino

$64(22,86)$

Raça

Branco

$268(95,71)$

Negro

$9(3,21)$

Pardo

$3(1,07)$

\section{Estado Civil}

Solteiro

$31(11,07)$

União Estável

$24(8,57)$

Casado

$163(58,21)$

Separado/Divorciado

$38(13,57)$

Viúvo

$24(8,57)$

Idade (anos)

31 a 42

$12(4,29)$

43 a 53

$70(25,00)$

54 a 64

$110(39,29)$

65 a 76

$65(23,21)$

77 a 90

$23(98,21)$

\section{Escolaridade}

Analfabeto

$20(7,14)$

Alfabetizado

$5(1,79)$

Fundamental Incompleto

$156(55,71)$

Fundamental Completo

$29(10,36)$

Médio Incompleto

$6(2,14)$

Médio Completo

$48(17,14)$

Superior Incompleto

$3(1,07)$

Superior Completo

$13(4,64)$ 
Fonte: Dados da pesquisa, 2017.

No que se refere aos dados relacionados aos fatores de risco e comorbidades associadas ao IAM (tabela 2), verificamos que $(41,43 \%)$ eram tabagista e $(2,86 \%)$ etilista. Pacientes que apresentaram Dislipidemia foram (17,86\%), Diabetes $(15,36 \%)$, Hipertensão Arterial Sistêmica (54,29\%) e Obesidade (2,86\%).

Tabela 2 - Fatores de risco e Comorbidades dos pacientes com Diagnósticos de Infarto Agudo do Miocárdio com Supra de segmento ST, Florianópolis-SC, Brasil, 2015 e 2016.

\section{Fatores de Risco}

Tabagista

Sim

Não

\section{Etilista}

Sim

Não

$$
\mathrm{n}=\mathbf{2 8 0}(\%)
$$

$116(41,43)$

$164(58,57)$

$8(2,86)$

$272(97,14)$

\section{Comorbidades}

\section{Dislipidemia}

Sim

Não

$$
230(82,14)
$$

\section{Diabete Melitus}

Sim

Não

Hipertensão Arterial Sistêmica

Sim

Não

$$
129(46,07)
$$

\section{Obesidade}

Sim

$$
8(2,86)
$$

Não

$272(94,14)$

Fonte: Dados da pesquisa, 2017. 
A Tabela 3 apresenta o tempo de espera dos pacientes desde o momento da classificação de gravidade até a angioplastia. Observa-se que o tempo médio de espera foi de 179 minutos; o tempo mínimo de 27 minutos e o tempo máximo de espera foi de 1204 minutos. Verificou-se que o tempo médio de espera está acima do preconizado pela V Diretriz da Sociedade Brasileira de Cardiologia sobre Tratamento do Infarto Agudo do Miocárdio com Supradesnível do Segmento ST, que é $\leq 90$ minutos.

Tabela 3 - DELTA T da Entrada no Centro de Referência até o Inicio da Angioplastia. Florianópolis-SC, Brasil, 2015 e 2016.

\begin{tabular}{cc}
\hline Variáveis & Tempo (minutos) \\
\hline Mín & 27 \\
Máx & 1204 \\
Média & 179 \\
Mediana & 114 \\
Std Dev & 199 \\
\hline
\end{tabular}

Fonte: Dados da pesquisa, 2017.

O tempo porta-balão foi comparado na tabela 4 de acordo com presença ou ausência de fatores de risco e comorbidades, entre homens e mulheres com Diagnósticos de Infarto Agudo do Miocárdio com Supra de Segmento ST. Os resultados indicam que não existem diferenças entre homens e mulheres no tempo porta-balão, homens (159 \pm 218 ) minutos versus $118 \pm 176$ minutos; $p=0,07)$. No que se refere aos fatores de risco, tempo porta-balão foi significativamente maior nos não tabagistas, quando comparado aos tabagistas (163 \pm 233 minutos versus $125 \pm 181$ minutos; $p=0,03$ ); já entre os pacientes etilistas, quando comparado aos não etilistas (85 \pm 151 minutos versus $157 \pm 205$ minutos; $p=0,19$ ), não apresentaram diferenças entre o tempo de espera. Não houve associação significativa entre o tempo portabalão com as comorbidades (Dislipidemia, Diabetes, Hipertensão Arterial Sistêmica e Obesidade) dos pacientes com Diagnósticos de Infarto Agudo do Miocárdio com Supra de segmento ST. 
Tabela 4 - Associação do tempo porta-balão com características, fatores de risco e comorbidades dos pacientes com Diagnósticos de Infarto Agudo do Miocárdio com Supra de segmento ST, Florianópolis-SC, Brasil, 2015 e 2016.

\begin{tabular}{lccc}
\hline Características & $\begin{array}{c}\text { Tempo Porta-Balão (minutos) } \\
\text { Médias }\end{array}$ & $\begin{array}{c}\text { Valor de } \mathbf{p} \\
\text { (IC 95\%) }\end{array}$ & $\mathbf{p}^{*}$ \\
\hline Sexo & & & \\
Masculino & 189 & $(159-218)$ & \\
Feminino & 147 & $(118-176)$ & 0,07 \\
\hline
\end{tabular}

Fatores de Risco

Tabagista

$\begin{array}{lrrr}\text { Sim } & 153 & (125-181) & \\ \text { Não } & 198 & (163-233) & 0,03\end{array}$

\section{Etilista}

$\begin{array}{lccc}\text { Sim } & 118 & (85-151) & \\ \text { Não } & 181 & (157-205) & 0,19\end{array}$

\section{Comorbidades}

\section{Dislipidemia}

$\begin{array}{llll}\text { Sim } & 157 & (115-200) & \\ \text { Não } & 184 & (157-211) & 0,20\end{array}$

\section{Diabete Melitus}

$\begin{array}{lccc}\text { Sim } & 154 & (113-195) & \\ \text { Não } & 184 & (157-211) & 0,18\end{array}$

Hipertensão Arterial Sistêmica

$\begin{array}{llll}\text { Sim } & 197 & (162-232) & \\ \text { Não } & 158 & (127-189) & 0,94\end{array}$

Obesidade

Sim

238

(179-459)

Não

177

(154-201)

0,80

Fonte: Dados da pesquisa, 2017.

* Valor de p calculado por teste t de Student não pareado. 
A tabela 5 mostra que o tempo médio porta-balão dos pacientes com IAM-ST é estatisticamente diferente de 90 minutos de espera, segundo a V Diretriz da Sociedade Brasileira de Cardiologia sobre Tratamento do Infarto Agudo do Miocárdio com Supradesnível do Segmento ST.

Tabela 5 - Média do tempo porta-balão dos pacientes com Diagnósticos de Infarto Agudo do Miocárdio com Supra de segmento ST, Florianópolis-SC, Brasil, 2015 e 2016.

\begin{tabular}{llccc}
\hline Tempo Porta-Balão & $(\mathrm{n})$ & Média & $($ IC95\%) & Valor de $\mathrm{p}^{*}$ \\
Minutos & 280 & 179 & $(\mathbf{1 5 6 - 2 0 2 )}$ & $\mathbf{0 , 0 0 0}$
\end{tabular}

* Valor de p calculado por teste t de Student (teste de hipótese para avaliar se o tempo médio porta-balão é estatisticamente diferente de 90 minutos N Diretriz da Sociedade Brasileira de Cardiologia sobre Tratamento do Infarto Agudo do Miocárdio com Supradesnível do Segmento ST. São Paulo: SBC, 2015. 121 p.)

\section{DISCUSSÃO}

Os resultados da pesquisa demonstraram que dos pacientes com diagnóstico IAM atendidos no centro de referência no ano de 2015 e 2016, não apresentou um aumento real de um ano para outro. Esta variação do número de pacientes atendidos em um serviço terciário de cardiologia pode sofrer alterações nem sempre significativas, incluindo a incidência de pacientes portadores de IAM 12,13. A idade, sexo e estado civil podem estar relacionados como um fator agravante para o IAM. Os resultados da pesquisa apontam a prevalência de IAM com supra de segmento ST em pacientes com idade média de 54 a 64 anos. Estudos mostram que na terceira idade a incidência desta patologia ocorre com maior frequência incluindo mulheres no climatério 2,14,15. Da mesma forma, quanto ao gênero, foi demonstrado maior frequência no sexo masculino, este fato se dá devido, a baixa procura a serviços de saúde de forma preventiva, não frequentando grupos de apoio, não aderindo ao tratamento medicamentoso de forma adequada e não procurando o serviço de saúde para acompanhamento 2,15 .

Ainda quanto à etnia os resultados da pesquisa mostraram um predomínio da etnia branca com relação a negros e pardos, resultado similares são descritos na literatura. O último censo do IBGE em 2010 estimou que dos 191 milhões de brasileiros, quase a metade são brancos, e menos de $10 \%$ são negros. A população 
de cor branca tem predomínio no Brasil, no entanto dados da literatura apontam que na raça negra há uma grande incidência de pessoas portadoras de HAS de difícil controle $^{2,16}$.

O perfil escolar, tem mostrado um resultado interessante, corroborando com outros estudos, mostrando que as pessoas com tempo de estudo $\leq 5$ anos, são mais afetadas pelo IAM. Estima-se que o déficit de conhecimento referente às comorbidades e o padrão de vida destas pessoas, estejam diretamente relacionados ao Infarto ${ }^{2,15}$.

As Unidades Básicas de Saúde (UBS) são responsáveis pela atenção primária à saúde, por vezes com baixa resolutividade, cabendo ao nível terciário à realização do diagnóstico e tratamento. A classe social com menor escolaridade normalmente apresenta menor poder econômico necessitando do serviço público para o controle e tratamento de doenças. Como a HAS não tem cura, apenas tratamento, muitas pessoas deixam de fazer o acompanhamento pelas UBS e acabam somente buscando o serviço de saúde no agravo da doença e, ou já com a doença instalada, no caso de um IAM. A população privilegiada economicamente faz uso de planos de saúde e tem acesso o aos serviços de saúde que podem apresentar maior resolutividade ${ }^{17}$.

As comorbidades e os maus hábitos de saúde estão relacionados diretamente ao infarto agudo do miocárdio com supradesnivelamento de segmento ST entre outras comorbidades. A HAS e a Dislipidemia são as principais comorbidades desencadeadoras deste processo e apontadas nesta pesquisa como predominantes, seguidos pelo DM. Outros estudos também mostraram que pacientes com síndrome coronariana apresentam HAS e dislipidemias. Os hábitos alimentares e de vida diária inadequados entre os quais o tabagismo, tem forte influência na síndrome coronariana 2,18 .

A síndrome coronariana, especificamente o IAMSST, necessita de um diagnóstico precoce, com tempo de dor inferior às $12 \mathrm{~h}$ e o acesso rápido a terapia de reperfusão, pois cada segundo que se perde maior o comprometido do músculo cardíaco. A literatura indica um tempo porta balão também denominado Delta $\mathrm{T}$, de 90 minutos como adequado para restauração do fluxo de sangue na artéria acometida. Os resultados da pesquisa apontam que o tempo porta balão é ainda bastante variável 
apresentando tempos alargados em alguns casos específicos. A média, no entanto permanece em três horas do tempo de entrada nos hospitais até o início da angioplastia, sendo superior ao recomendado 3,19,20.

$\mathrm{O}$ atendimento ao Infarto, principalmente ao infarto com o supra ST, onde o tempo resposta é primordial, o atendimento deve ser ágil, organizado, unidirecional e principalmente seguindo os protocolos e realizações de feedback quanto ao atendimento. $\mathrm{O}$ enfermeiro tem papel fundamental desde a entrada do paciente na instituição, identificação/resolução do problema e alta, para tal fato capacitação e o conhecimento são imperativos sendo essencial para o cuidado ${ }^{21,22}$. Para a criação e atualização dos protocolos, o enfermeiro precisa se manter atualizado e capacitado para tal, o mesmo deve manter a educação continuada em seu local de trabalho através de cronogramas, planejamentos e avaliação de funcionamento dos mesmos ,23,24. A necessidade de conhecer e identificar precocemente o IAMCSST exige aprimoramento técnico diário através do estudo específico sobre eletrocardiograma, por ser este o exame primordial da detecção precoce do infarto ${ }^{25}$.

Medidas específicas poderão ser adotadas a fim de aumentar a efetividade do atendimento visando à redução do tempo porta balão. Entre as medidas estão os protocolos e adoção de um fluxo previamente determinado pela instituição de saúde envolvendo toda a equipe de saúde, que devera ser continuamente reavaliado buscando diminuir as não conformidades que poderão ser encontradas. ${ }^{26,27,28}$.

\section{CONLUSÃO}

Com o estudo, foi possível traçar o perfil epidemiológico das pessoas mais afetadas pelo IAMCSST na mesorregião da grande Florianópolis, corroborando com outros estudos, quanto à etnia, sexo, escolaridade, idade. Tratando sobre IAM vários fatores podem desencadear o mesmo, o estilo de vida e comportamento das pessoas influencia diretamente no processo de saúde/doença. Quanto ao tempo resposta, devemos levar em consideração a superlotação dos hospitais públicos e por vezes a falta de profissionais, materiais e equipamentos. Sabendo que o tempo resposta é o principal passo para a reperfusão da área isquêmica no infarto com supradesnível, é necessário interrogar os processos de trabalho objetivando alcançar índices 
aceitáveis e compatíveis como os recomendados pela Sociedade Brasileira de Cardiologia. O enfermeiro deve cada vez mais se capacitar, por se situar como líder da equipe de enfermagem e responsável por planejar e implementar a assistência de enfermagem, cabendo ao mesmo o processo de educação continuada e avaliação diária das boas práticas em enfermagem. A SAE, também se torna essencial para a assistência de enfermagem a este paciente, uma vez que possibilita elencar um plano de cuidados com base nos dados coletados na história, exame físico e diagnóstico de enfermagem. Como resultado a assistência ao paciente se torna mais eficaz e segura, promovendo maior conforto e qualidade do atendimento, refletindo em uma recuperação com tempo de internação por vezes mais reduzido. De outro modo também é necessário identificar se $o$ atendimento pré-hospitalar pode aprimorar suas práticas e protocolos baseado na evidência, como sendo um elo importante na redução do tempo porta-balão. Com vistas a qualificar os serviços, a implantação ou reavaliação dos processos de trabalho especificamente os relacionados a assistência ao paciente favorecem a qualificação e eficiência do serviço prestado a população.

\section{REFERÊNCIA}

1. Ribeiro RM, Cesarino CB, de Cássia HMRR, Rodrigues CC, Bertolin DC, Pinto MH, Becária LM. Perfil de emergências clínicas no departamento de emergência de um hospital de ensino. Reme: Revista Mineira de Enfermagem, v. 18, n. 3, p. 533-538, 2014. GN1 Genesis Network. Disponível em: Http://dx.doi.org/10.5935/1415-2762.20140039. Acesso em: jun. 2018.

2. $D a$ Costa FAS, Parente $F L$, Farias $M S$, Parente FL, Francelino PC, Bezerra LTL. SANARE, Sobral - v.17 n.02, p.66-73, 2018. Disponível em: https://sanare.emnuvens.com.br/sanare/article/view/1263. Acesso em: ago. 2019

3. Silva AJS, Guimarães CSS, Reis JÁ. Perfil de pacientes internados com diagnóstico de síndrome coronariana aguda. Rev Soc Bras Clin Med. v. 16(2), 
2018.

Disponível

em:

http://www.sbcm.org.br/ojs3/index.php/rsbcm/article/view/341/309. Acesso em ago. 2019.

4. Sociedade Brasileira de Cardiologia. V Diretriz da Sociedade Brasileira de Cardiologia sobre Tratamento do Infarto Agudo do Miocárdio com Supradesnível do Segmento ST. São Paulo: SBC, 2015. 121 p.

5. Marino BCA, Marcolino MS, Reis Júnior R dos S, França ALN, Passos PFO, et al. Perfil Epidemiológico e Indicadores de Qualidade em Pacientes com Síndrome Coronariana Aguda na Região Norte de Minas Gerais - Projeto Minas Telecardio 2 Arq. Bras. Cardiol. 107(2):106-115, 2016. Disponível em: http://www.scielo.br/scielo.php?pid=S0066782X2016005017102\&script=sci_arttext\&tIng=pt. Acesso em: ago. 2019

6. Araújo DF, Araújo ERM, Silva MRV, Silva NC, Guimarães MSO, Neta FLA. Perfil clínico e epidemiológico de pacientes com síndrome coronariana aguda. Revista de Enfermagem da UFPI, Teresina, v. 3 (2), p.78-84, 2014. Disponível em: http://www.ojs.ufpi.br/index.php/reufpi/article/view/1895/pdf. Acesso em jun 2018.

7. DATASUS. Departamento de informática do SUS. Infarto agudo do miocárdio é primeira causa de mortes no País, revela dados do DATASUS. [Internet]. 2014. Disponível em: http://tabnet.datasus.gov.br/cgi/tabcgi.exe?sim/cnv/obt10uf.def . Acesso em: mar. 2016

8. BRASIL. Ministério da Saúde. Ministério da Saúde (Ed.). Manual Instrutivo da Rede de Atenção às Urgências e Emergências no Sistema Único de Saúde (SUS). Brasília: MS, $2013 . \quad 86 \quad$ p. Disponível em: http://bvsms.saude.gov.br/bvs/publicacoes/manual_instrutivo_rede_atencao_urg encias.pdf. Acesso em: set. 2017

9. Pertsew PE, Perozin M, Chaves PLL. Gerenciamento do protocolo de dor torácica no setor de emergência. Rev Soc Bras Clin Me.16(2):77-9, 2018. Disponível em: 
http://www.sbcm.org.br/ojs3/index.php/rsbcm/article/view/335/303. Acesso em ago. 2019.

10. Conselho Nacional de Saúde (Brasil). Resolução no 466, de 12 de dezembro de 2012. Brasília, 2012. Disponível em: http://www.conselho. saude.gov.br/web_comissoes/conep/index.html. Acesso em: ago. 2017.

11. Framingham Heart Study. Cardiovascular Disease (10-year risk) Framingham (USA). Disponível em: https://www.framinghamheartstudy.org/riskfunctions/cardiovasculardisease/10-year-risk.php. Acesso em ago. 2017.

12. Rocha AS, Araújo MP, Volscham A, Carvalho LAF, Ribeira A, Mesquita ET. Evidência de Melhora na Qualidade do Cuidado Assistencial no Infarto Agudo do Miocárdio. Arquivos Brasileiros de Cardiologia, São Paulo, v. 94(6), p.726-729, 2010. Disponível em: http://www.scielo.br/pdf/abc/v94n6/aop04310.pdf. Acesso em: 06 mai. 2017.

13. Marcolino MS, Brant LCC, Araujo JG, Nascimento BR, Castro LRA, Martins P, et al. Implementation of the Myocardial Infarction System of Care in City of Belo Horizonte, Brazil. Arquivos Brasileiros de Cardiologia,100(4), p.307-314, 2013. GN1 Genesis Network. Disponível em: http://dx.doi.org/10.5935/abc.20130054. Acesso em: 06 mai. 2017.

14. De Melo JB, Campos RCA, Carvalho PC, Meireles MF, Andrade MVG, et al. Fatores de Risco Cardiovasculares em Mulheres Climatéricas com Doença Arterial Coronariana. Int J Cardiovasc Sci. 31(1)4-11 2018. Disponível em: http://www.scielo.br/pdf/ijcs/v31n1/pt_2359-4802-ijcs-20170056.pdf. Acesso em: jan 2019

15. Marino BCA, Marcolino MS, Júnior RSR, França ALN, Passos PFO, Lemos TR, et al. Epidemiological Profile and Quality Indicators in Patients with Acute Coronary Syndrome in Northern Minas Gerais - Minas Telecardio 2 Project. Arquivos Brasileiros de Cardiologia, 107(2):106-115, 2016. Disponível em: 
http://www.scielo.br/pdf/abc/v107n2/pt_0066-782X-abc-20160095.pdf. Acesso em: ago. 2017.

16. Brunori, EHDR, Lopes CT, Cavalcante AMRZ, Santos VB, Lopes JL, Barros ALBL. Association of cardiovascular risk factors with the different presentations of acute coronary syndrome. Revista Latino-americana de Enfermagem, 22(4):53846, 2014. Disponível em: http://www.scielo.br/pdf/rlae/v22n4/0104-1169-rlae-2204-00538.pdf. Acesso em set. 2018.

17. Chagas MIO, Farias CMT, Teófilo FKS, Cavalcante ASP. Acesso dos usuários aos serviços de saúde na estratégia saúde da família: percepção dos enfermeiros. Revista Aps, Sobral, v. 17, n. 3, p.280-290, set. 2014. Disponível em: https://aps.ufjf.emnuvens.com.br/aps/article/view/2183. Acesso em jun. 2017.

18. Oliveira $\mathrm{CH}$, Oliveira HE, Moreira DM, Carvalho AAP. Fatores associados ao óbito intra-hospitalar em pacientes internados por infarto agudo do miocárdio.Arquivo Catarinense de Medicina, São José, v. 45, n. 4, p.28-40, dez. 2016. Disponível em: $\quad$ http://www.acm.org.br/acm/seer/index.php/arquivos/article/view/135/112. Acesso em: 2018.

19. Bastos AS, Beccaria LM, Contrin LM, Cesarino CB. Time of arrival of patients with acute myocardial infarction to the emergency department. Rev Bras Cir Cardiovasc. 27(3):411-8, 2012.

Disponível em: http://www.scielo.br/pdf/rbccv/v27n3/v27n3a12.pdf. Acesso em out. 2018

20. Figueiredo AE, Siebel AL, Luce DC, Schneider I. Determinação do tempo de apresentação a emergência de pacientes com infarto agudo do miocárdio. Revista Enfermagem UFSM, Santa Maria, v. 3 (1), p.93-101, 2013. Disponível em: https://periodicos.ufsm.br/index.php/reufsm/article/view/7422. Acesso em: 07 maio 2017. 
21. Caveião C, Santos RB, Montezeli JH, Visentin A, Brey C, Oliveira VBCA. Dor torácica: atuação do enfermeiro em um pronto atendimento de um hospital escola. R Enferm. Cent. O Min, jan./abr. 4(1),921-928, 2014. Disponível em: http://www.seer.ufsj.edu.br/index.php/recom/article/view/427/567. Acesso em: mar. 2018.

22. Maier GSO, Martins EAP. Assistência ao paciente com síndrome coronariana aguda segundo indicadores de qualidade. Rev Bras Enferm [Internet].69(4):75764, 2016. Disponível em: http://www.scielo.br/pdf/reben/v69n4/0034-7167-reben69-04-0757.pdf. Acesso em: ago. 2019.

23. Pereira L, Cordeiro RB, Marques SCA, Biernaski AE, Miranda AKO. Infarto agudo do miocárdio: Atuação do profissional enfermeiro. Vitrine Prod. Acad., Curitiba, v.6, n.1, p.260-281, jan/dez. 2018

24. PAP, Oliveira WA. Avaliação de protocolo de dor torácica no Hospital do coração do Brasil. Ver. Cient. Sena Aires, 5(2):136-149, 2016. Disponível em: http://revistafacesa.senaaires.com.br/index.php/revisa/article/view/265/138. Acesso em: fev. 2019.

25. Thaler MS. ECG essencial: Eletrocardiograma na prática diária. 7. ed. Porto Alegre: Artmed, 2013. 332 p.

26. Makdisse M, Katz M, Corrêa AG, Forlenza LMA, Perin MA, Junior FSB, et al. Efeito da implementação de um protocolo assistencial de infarto agudo do miocárdio sobre os indicadores de qualidade. Einstein, 11(3):357-63, 2013. Disponível em: http://www.scielo.br/pdf/eins/v11n3/a16v11n3.pdf. Acesso em: mar. 2019.

27. Campos HAB, Moreira MVF, Alves EE, Wang R, Bedeti ACM, et al. Impacto da adoção de processos de trabalho hospitalares na redução do tempo porta-balão. Rev Bras Cardiol Invasiva. 25(1-4):7-11, 2017. Disponível em: https://jotci.org/wp- 
content/uploads/articles_xml/0104-1843-rbci-25-01-0007/0104-1843-rbci-25-010007.pdf. Acesso em: ago. 2019

28. Dias PAP, Oliveira WA. Avaliação do protocolo de dor torácica no hospital do coração do Brasil. Rev. Cient. Sena Aires. 5(2): 136-49, 2016. Disponível em: http://revistafacesa.senaaires.com.br/index.php/revisa/article/view/265/138. Acesso em: ago. 2019. 